\title{
Characterizations for the Riesz potential and its commutators on generalized Orlicz-Morrey spaces
}

\author{
Fatih Deringoz ${ }^{1 *}$, Vagif S Guliyev ${ }^{1,2}$ and Sabir G Hasanov ${ }^{3}$
}

\section{"Correspondence:}

deringoz@hotmail.com

'Department of Mathematics, Ahi

Evran University, Kirsehir, Turkey

Full list of author information is

available at the end of the article

\begin{abstract}
In the present paper, we shall give a characterization for the Spanne and Adams type boundedness of the Riesz potential and its commutators on the generalized Orlicz-Morrey spaces, respectively. Also we give criteria for the weak versions of Spanne and Adams type boundedness of the Riesz potential on the generalized Orlicz-Morrey spaces. In all the cases the conditions for the boundedness are given in terms of Zygmund type integral inequalities involving the Young function $\Phi(u)$ and the function $\varphi(x, r)$ defining the space.
\end{abstract}

MSC: 42B20; 42B25; 32A37; 46E30

Keywords: generalized Orlicz-Morrey space; Riesz potential; commutator; BMO

\section{Introduction}

As is well known, Morrey spaces are widely used to investigate the local behavior of solutions to second order elliptic partial differential equations (PDE). Recall that the classical Morrey spaces $\mathcal{M}^{p, \lambda}\left(\mathbb{R}^{n}\right)$ are defined by

$$
\mathcal{M}^{p, \lambda}\left(\mathbb{R}^{n}\right)=\left\{f \in L_{\mathrm{loc}}^{p}\left(\mathbb{R}^{n}\right):\|f\|_{\mathcal{M}^{p, \lambda}}:=\sup _{x \in \mathbb{R}^{n}, r>0} r^{-\frac{\lambda}{p}}\|f\|_{L^{p}(B(x, r))}<\infty\right\},
$$

where $0 \leq \lambda \leq n, 1 \leq p<\infty . \mathcal{M}^{p, \lambda}\left(\mathbb{R}^{n}\right)$ was an expansion of $L^{p}\left(\mathbb{R}^{n}\right)$ in the sense that $\mathcal{M}^{p, 0}\left(\mathbb{R}^{n}\right)=L^{p}\left(\mathbb{R}^{n}\right)$ and $\mathcal{M}^{p, n}\left(\mathbb{R}^{n}\right)=L^{\infty}\left(\mathbb{R}^{n}\right)$.

Here and everywhere in the sequel $B(x, r)$ is the ball in $\mathbb{R}^{n}$ of radius $r$ centered at $x$ and $|B(x, r)|=v_{n} r^{n}$ is its Lebesgue measure, where $v_{n}$ is the volume of the unit ball in $\mathbb{R}^{n}$.

By $W \mathcal{M}^{p, \lambda}\left(\mathbb{R}^{n}\right)$ we denote the weak Morrey space defined as the set of functions $f$ in the local weak space $W L_{\mathrm{loc}}^{p}\left(\mathbb{R}^{n}\right)$ for which

$$
\|f\|_{W \mathcal{M} p, \lambda}=\sup _{x \in \mathbb{R}^{n}, r>0} r^{-\frac{\lambda}{p}}\|f\|_{W L^{p}(B(x, r))}<\infty
$$

The spaces $\mathcal{M}^{p, \varphi}\left(\mathbb{R}^{n}\right)$ defined by the norm

$$
\|f\|_{\mathcal{M}^{p, \varphi}}=\sup _{x \in \mathbb{R}^{n}, r>0} \varphi(x, r)^{-1}|B(x, r)|^{-\frac{1}{p}}\|f\|_{L^{p}(B(x, r))}
$$

(c) 2016 Deringoz et al. This article is distributed under the terms of the Creative Commons Attribution 4.0 International License (http://creativecommons.org/licenses/by/4.0/), which permits unrestricted use, distribution, and reproduction in any medium, provided you give appropriate credit to the original author(s) and the source, provide a link to the Creative Commons license, and indicate if changes were made. 
with a function $\varphi$ positive and measurable on $\mathbb{R}^{n} \times(0, \infty)$ are known as generalized Morrey spaces (see, for example, [1-3]). Also by $W \mathcal{M}^{p, \varphi}\left(\mathbb{R}^{n}\right)$ we denote the weak generalized Morrey space of all functions $f \in W L_{\mathrm{loc}}^{p}\left(\mathbb{R}^{n}\right)$ for which

$$
\|f\|_{W \mathcal{M}^{p, \varphi}}=\sup _{x \in \mathbb{R}^{n}, r>0} \varphi(x, r)^{-1}|B(x, r)|^{-\frac{1}{p}}\|f\|_{W L^{p}(B(x, r))}<\infty .
$$

The Orlicz space was first introduced by Orlicz in $[4,5]$ as a generalization of Lebesgue spaces $L^{p}$. Since then this space has been one of the important functional frames in mathematical analysis, and especially in real and harmonic analysis. Orlicz space is also an appropriate substitute for $L^{1}$ space when $L^{1}$ space does not work. For example, the HardyLittlewood maximal operator

$$
M f(x)=\sup _{r>0} \frac{1}{|B(x, r)|} \int_{B(x, r)}|f(y)| d y
$$

is bounded on $L^{p}$ for $1<p<\infty$, but not on $L^{1}$, but using Orlicz spaces, we can investigate the boundedness of the maximal operator near $p=1$, see [6, 7] and [8] for more precise statements.

A natural step in the theory of functions spaces was to study Orlicz-Morrey spaces $\mathcal{M}^{\Phi, \varphi}\left(\mathbb{R}^{n}\right)$, where the 'Morrey type measuring' of the regularity of functions is realized with respect to the Orlicz norm over balls instead of the Lebesgue one. Such spaces were first introduced and studied by Nakai [9]. Then another kind of Orlicz-Morrey spaces were introduced by Sawano et al. [10]. Our definition of Orlicz-Morrey spaces introduced in [11] and used here is different from that of [9] and [10].

Let $0<\alpha<n$. The Riesz potential operator $I_{\alpha}$ is defined by

$$
I_{\alpha} f(x)=\int_{\mathbb{R}^{n}} \frac{f(y)}{|x-y|^{n-\alpha}} d y .
$$

The commutators generated by $b \in L_{\text {loc }}^{1}\left(\mathbb{R}^{n}\right)$ and the operator $I_{\alpha}$ are defined by

$$
\left[b, I_{\alpha}\right] f(x)=\int_{\mathbb{R}^{n}} \frac{b(x)-b(y)}{|x-y|^{n-\alpha}} f(y) d y .
$$

The operator $\left|b, I_{\alpha}\right|$ is defined by

$$
\left|b, I_{\alpha}\right| f(x)=\int_{\mathbb{R}^{n}} \frac{|b(x)-b(y)|}{|x-y|^{n-\alpha}} f(y) d y .
$$

The classical result by Hardy-Littlewood-Sobolev states that the operator $I_{\alpha}$ is of weak type $(p, n p /(n-\alpha p))$ if $1 \leq p<n / \alpha$ and of strong type $(p, n p /(n-\alpha p))$ if $1<p<n / \alpha$.

Around the 1970s, the Hardy-Littlewood-Sobolev inequality was extended from Lebesgue spaces to Morrey spaces. As stated in [12], Spanne proved the following result.

Theorem 1.1 (Spanne, but published by Peetre [12]) Let $0<\alpha<n, 1 \leq p<\frac{n}{\alpha}, 0<\lambda<$ $n-\alpha p$. Moreover, let $\frac{1}{p}-\frac{1}{q}=\frac{\alpha}{n}$ and $\frac{\lambda}{p}=\frac{\mu}{q}$. Then, for $p>1$, the operator $I_{\alpha}$ is bounded from $\mathcal{M}^{p, \lambda}\left(\mathbb{R}^{n}\right)$ to $\mathcal{M}^{q, \mu}\left(\mathbb{R}^{n}\right)$ and for $p=1, I_{\alpha}$ is bounded from $\mathcal{M}^{1, \lambda}\left(\mathbb{R}^{n}\right)$ to $W \mathcal{M}^{q, \mu}\left(\mathbb{R}^{n}\right)$. 
Later on, a stronger result was obtained by Adams [13], and refined by Chiarenza and Frasca [14].

Theorem 1.2 (Adams [13]) Let $0<\alpha<n, 1 \leq p<\frac{n}{\alpha}, 0<\lambda<n-\alpha p$ and $\frac{1}{p}-\frac{1}{q}=\frac{\alpha}{n-\lambda}$. Then, for $p>1$, the operator $I_{\alpha}$ is bounded from $\mathcal{M}^{p, \lambda}\left(\mathbb{R}^{n}\right)$ to $\mathcal{M}^{q, \lambda}\left(\mathbb{R}^{n}\right)$ and for $p=1, I_{\alpha}$ is bounded from $\mathcal{M}^{1, \lambda}\left(\mathbb{R}^{n}\right)$ to $W \mathcal{M}^{q, \lambda}\left(\mathbb{R}^{n}\right)$.

Guliyev [1] (see also, $[15,16])$ extended the results of Spanne and Adams from Morrey spaces to generalized Morrey spaces. Later on, Spanne type results were obtained by Guliyev et al. [17] under a weaker condition. These results can be summarized as follows.

Theorem 1.3 ([17]) Let $0<\alpha<n, 1 \leq p<\frac{n}{\alpha}, \frac{1}{q}=\frac{1}{p}-\frac{\alpha}{n}$ and $\left(\varphi_{1}, \varphi_{2}\right)$ satisfy the condition

$$
\int_{r}^{\infty} \frac{\operatorname{essinf}_{t<s<\infty} \varphi_{1}(x, s) s^{\frac{n}{p}}}{t^{\frac{n}{q}+1}} d t \leq C \varphi_{2}(x, r)
$$

where $C$ does not depend on $x$ and $r$. Then $I_{\alpha}$ is bounded from $\mathcal{M}^{p, \varphi_{1}}\left(\mathbb{R}^{n}\right)$ to $\mathcal{M}^{q, \varphi_{2}}\left(\mathbb{R}^{n}\right)$ for $p>1$ and from $\mathcal{M}^{p, \varphi_{1}}\left(\mathbb{R}^{n}\right)$ to $W \mathcal{M}^{q, \varphi_{2}}\left(\mathbb{R}^{n}\right)$ for $p \geq 1$.

Theorem 1.4 ([1]) Let $1 \leq p<\infty, 0<\alpha<\frac{n}{p}$ and let $\varphi(x, t)$ satisfy the conditions

$$
\sup _{r<t<\infty} \frac{\operatorname{essinf}_{t<s<\infty} \varphi(x, s) s^{\frac{n}{p}}}{t^{\frac{n}{p}}} \leq C \varphi(x, r)
$$

and

$$
t^{\alpha} \varphi(x, t)+\int_{t}^{\infty} r^{\alpha} \varphi(x, r) \frac{d r}{r} \leq C \varphi(x, t)^{\frac{p}{q}}
$$

where $q>p$ and $C$ does not depend on $x \in \mathbb{R}^{n}$ and $t>0$. Suppose also that, for almost every $x \in \mathbb{R}^{n}$, the function $\varphi(x, r)$ fulfills the condition

there exist an $a=a(x)>0$ such that $\varphi(x, \cdot):[0, \infty] \rightarrow[a, \infty)$ is surjective.

Then, for $p>1$, the operator $I_{\alpha}$ is bounded from $\mathcal{M}^{p, \varphi}\left(\mathbb{R}^{n}\right)$ to $\mathcal{M}^{q, \varphi^{\frac{p}{q}}}\left(\mathbb{R}^{n}\right)$ and for $p \geq 1$ the operator $I_{\alpha}$ is bounded from $\mathcal{M}^{p, \varphi}\left(\mathbb{R}^{n}\right)$ to $W \mathcal{M}^{q, \varphi^{\frac{1}{q}}}\left(\mathbb{R}^{n}\right)$.

It is well known that commutators of classical operators of harmonic analysis play an important role in various topics of analysis and PDE.

As an application of the above results, the boundedness of $\left[b, I_{\alpha}\right]$ on generalized Morrey spaces is investigated in $[17,18]$.

The boundedness of $I_{\alpha}$ from Orlicz space $L^{\Phi}\left(\mathbb{R}^{n}\right)$ to the corresponding another Orlicz space $L^{\Psi}\left(\mathbb{R}^{n}\right)$ was studied in [8]. There were given necessary and sufficient conditions for the boundedness of $I_{\alpha}$ from $L^{\Phi}\left(\mathbb{R}^{n}\right)$ to $L^{\Psi}\left(\mathbb{R}^{n}\right)$ and also from $L^{\Phi}\left(\mathbb{R}^{n}\right)$ to the weak Orlicz space $W L^{\Psi}\left(\mathbb{R}^{n}\right)$.

In this paper, we shall give a characterization for the Spanne and Adams type boundedness of the Riesz potential and its commutators on generalized Orlicz-Morrey spaces, 
respectively. Also we give criteria for the weak versions of Spanne and Adams type boundedness of the Riesz potential on the generalized Orlicz-Morrey spaces.

By $A \lesssim B$ we mean that $A \leq C B$ with some positive constant $C$ independent of appropriate quantities. If $A \lesssim B$ and $B \lesssim A$, we write $A \approx B$ and say that $A$ and $B$ are equivalent.

\section{Preliminaries}

\subsection{On Young functions and Orlicz spaces}

We recall the definition of Young functions.

Definition 2.1 A function $\Phi:[0, \infty) \rightarrow[0, \infty]$ is called a Young function if $\Phi$ is convex, left-continuous, and we have $\lim _{r \rightarrow+0} \Phi(r)=\Phi(0)=0$ and $\lim _{r \rightarrow \infty} \Phi(r)=\infty$.

From the convexity and $\Phi(0)=0$ it follows that any Young function is increasing. If there exists $s \in(0, \infty)$ such that $\Phi(s)=\infty$, then $\Phi(r)=\infty$ for $r \geq s$. The set of Young functions such that

$$
0<\Phi(r)<\infty \text { for } 0<r<\infty
$$

will be denoted by $\mathcal{Y}$. If $\Phi \in \mathcal{Y}$, then $\Phi$ is absolutely continuous on every closed interval in $[0, \infty)$ and bijective from $[0, \infty)$ to itself.

For a Young function $\Phi$ and $0 \leq s \leq \infty$, let

$$
\Phi^{-1}(s)=\inf \{r \geq 0: \Phi(r)>s\} .
$$

If $\Phi \in \mathcal{Y}$, then $\Phi^{-1}$ is the usual inverse function of $\Phi$. We note that

$$
\Phi\left(\Phi^{-1}(r)\right) \leq r \leq \Phi^{-1}(\Phi(r)) \quad \text { for } 0 \leq r<\infty .
$$

It is well known that

$$
r \leq \Phi^{-1}(r) \widetilde{\Phi}^{-1}(r) \leq 2 r \quad \text { for } r \geq 0,
$$

where $\widetilde{\Phi}(r)$ is defined by

$$
\widetilde{\Phi}(r)= \begin{cases}\sup \{r s-\Phi(s): s \in[0, \infty)\}, & r \in[0, \infty), \\ \infty, & r=\infty\end{cases}
$$

A Young function $\Phi$ is said to satisfy the $\Delta_{2}$-condition, denoted also as $\Phi \in \Delta_{2}$, if

$$
\Phi(2 r) \leq k \Phi(r) \quad \text { for } r>0
$$

for some $k>1$. If $\Phi \in \Delta_{2}$, then $\Phi \in \mathcal{Y}$. A Young function $\Phi$ is said to satisfy the $\nabla_{2}$ condition, denoted also by $\Phi \in \nabla_{2}$, if

$$
\Phi(r) \leq \frac{1}{2 k} \Phi(k r), \quad r \geq 0
$$

for some $k>1$. 
Definition 2.2 (Orlicz space) For a Young function $\Phi$, the set

$$
L^{\Phi}\left(\mathbb{R}^{n}\right)=\left\{f \in L_{\mathrm{loc}}^{1}\left(\mathbb{R}^{n}\right): \int_{\mathbb{R}^{n}} \Phi(k|f(x)|) d x<\infty \text { for some } k>0\right\}
$$

is called Orlicz space. If $\Phi(r)=r^{p}, 1 \leq p<\infty$, then $L^{\Phi}\left(\mathbb{R}^{n}\right)=L^{p}\left(\mathbb{R}^{n}\right)$. If $\Phi(r)=0(0 \leq r \leq 1)$ and $\Phi(r)=\infty(r>1)$, then $L^{\Phi}\left(\mathbb{R}^{n}\right)=L^{\infty}\left(\mathbb{R}^{n}\right)$. The space $L_{\text {loc }}^{\Phi}\left(\mathbb{R}^{n}\right)$ is defined as the set of all functions $f$ such that $f \chi_{B} \in L^{\Phi}\left(\mathbb{R}^{n}\right)$ for all balls $B \subset \mathbb{R}^{n}$.

$L^{\Phi}\left(\mathbb{R}^{n}\right)$ is a Banach space with respect to the norm

$$
\|f\|_{L^{\Phi}}=\inf \left\{\lambda>0: \int_{\mathbb{R}^{n}} \Phi\left(\frac{|f(x)|}{\lambda}\right) d x \leq 1\right\} .
$$

We note that

$$
\int_{\mathbb{R}^{n}} \Phi\left(\frac{|f(x)|}{\|f\|_{L^{\Phi}}}\right) d x \leq 1
$$

Lemma 2.3 ([11]) For a Young function $\Phi$ and $B=B(x, r)$, the following inequality is valid:

$$
\|f\|_{L^{1}(B)} \leq 2|B| \Phi^{-1}\left(|B|^{-1}\right)\|f\|_{L^{\Phi}(B)}
$$

where $\|f\|_{L^{\Phi}(B)}=\left\|f \chi_{B}\right\|_{L^{\Phi}}$.

By elementary calculations we have the following.

Lemma 2.4 Let $\Phi$ be a Young function and $B$ a set in $\mathbb{R}^{n}$ with finite Lebesgue measure. Then

$$
\left\|\chi_{B}\right\|_{L^{\Phi}}=\frac{1}{\Phi^{-1}\left(|B|^{-1}\right)} .
$$

\subsection{Generalized Orlicz-Morrey space}

Various versions of generalized Orlicz-Morrey spaces were introduced in $[9,10]$ and [11]. We used the definition of [11] which runs as follows.

Definition 2.5 Let $\varphi(x, r)$ be a positive measurable function on $\mathbb{R}^{n} \times(0, \infty)$ and $\Phi$ any Young function. We denote by $\mathcal{M}^{\Phi, \varphi}\left(\mathbb{R}^{n}\right)$ the generalized Orlicz-Morrey space, the space of all functions $f \in L_{\text {loc }}^{\Phi}\left(\mathbb{R}^{n}\right)$ for which

$$
\|f\|_{\mathcal{M}^{\Phi, \varphi}}=\sup _{x \in \mathbb{R}^{n}, r>0} \varphi(x, r)^{-1} \Phi^{-1}\left(|B(x, r)|^{-1}\right)\|f\|_{L^{\Phi}(B(x, r))}<\infty .
$$

The following theorem and lemma play a key role in our main results.

Theorem 2.6 ([11]) Let $\Phi \in \mathcal{Y}$, the functions $\varphi$ and $\Phi$ satisfy the condition

$$
\sup _{r<t<\infty} \Phi^{-1}\left(|B(x, t)|^{-1}\right) \underset{t<s<\infty}{\operatorname{essinf}} \frac{\varphi(x, s)}{\Phi^{-1}\left(|B(x, s)|^{-1}\right)} \leq C \varphi(x, r),
$$


where $C$ does not depend on $x$ and $r$. Then the maximal operator $M$ is bounded from $\mathcal{M}^{\Phi, \varphi}\left(\mathbb{R}^{n}\right)$ to $\mathcal{M}^{\Phi, \varphi}\left(\mathbb{R}^{n}\right)$ for $\Phi \in \nabla_{2}$.

A function $\varphi:(0, \infty) \rightarrow(0, \infty)$ is said to be almost increasing (resp. almost decreasing) if there exists a constant $C>0$ such that

$$
\varphi(r) \leq C \varphi(s) \quad(\text { resp. } \varphi(r) \geq C \varphi(s)) \quad \text { for } r \leq s
$$

For a Young function $\Phi$, we denote by $\mathcal{G}_{\Phi}$ the set of all almost decreasing functions $\varphi$ : $(0, \infty) \rightarrow(0, \infty)$ such that $t \in(0, \infty) \mapsto \frac{1}{\Phi^{-1}\left(v_{n}^{-1} t^{-n}\right)} \varphi(t)$ is almost increasing.

Lemma 2.7 Let $B_{0}:=B\left(x_{0}, r_{0}\right)$. If $\varphi \in \mathcal{G}_{\Phi}$, then there exists $C>0$ such that

$$
\frac{1}{\varphi\left(r_{0}\right)} \leq\left\|\chi_{B_{0}}\right\|_{\mathcal{M}^{\Phi, \varphi}} \leq \frac{C}{\varphi\left(r_{0}\right)}
$$

Proof Let $B=B(x, r)$ denote an arbitrary ball in $\mathbb{R}^{n}$. By the definition and Lemma 2.4 , it is easy to see that

$$
\begin{aligned}
\left\|\chi_{B_{0}}\right\|_{\mathcal{M}^{\Phi, \varphi}} & =\sup _{x \in \mathbb{R}^{n}, r>0} \varphi(r)^{-1} \Phi^{-1}\left(|B|^{-1}\right) \frac{1}{\Phi^{-1}\left(\left|B \cap B_{0}\right|^{-1}\right)} \\
& \geq \varphi\left(r_{0}\right)^{-1} \Phi^{-1}\left(\left|B_{0}\right|^{-1}\right) \frac{1}{\Phi^{-1}\left(\left|B_{0} \cap B_{0}\right|^{-1}\right)}=\frac{1}{\varphi\left(r_{0}\right)} .
\end{aligned}
$$

Now if $r \leq r_{0}$, then $\varphi\left(r_{0}\right) \leq C \varphi(r)$ and

$$
\varphi(r)^{-1} \Phi^{-1}\left(|B|^{-1}\right)\left\|\chi_{B_{0}}\right\|_{L^{\Phi}(B)} \leq \frac{1}{\varphi(r)} \leq \frac{C}{\varphi\left(r_{0}\right)} .
$$

On the other hand if $r \geq r_{0}$, then $\frac{\varphi\left(r_{0}\right)}{\Phi^{-1}\left(\left|B_{0}\right|^{-1}\right)} \leq C \frac{\varphi(r)}{\Phi^{-1}\left(|B|^{-1}\right)}$ and

$$
\varphi(r)^{-1} \Phi^{-1}\left(|B|^{-1}\right)\left\|\chi_{B_{0}}\right\|_{L^{\Phi}(B)} \leq \frac{C}{\varphi\left(r_{0}\right)}
$$

This completes the proof.

\section{Spanne type results for $I_{\alpha}$ in the spaces $\mathcal{M}^{\Phi, \varphi}$}

We recall that, for functions $\Phi$ and $\Psi$ from $[0, \infty)$ into $[0, \infty]$, the function $\Psi$ is said to dominate $\Phi$ globally if there exists a positive constant $c$ such that $\Phi(s) \leq \Psi(c s)$ for all $s \geq 0$.

In the theorems below we also use the notation

$$
\widetilde{\Psi}_{P}(s)=\int_{0}^{s} r^{P^{\prime}-1}\left(\mathcal{B}_{P}^{-1}\left(r^{P^{\prime}}\right)\right)^{P^{\prime}} d r
$$

where $1<P \leq \infty$ and $\widetilde{\Psi}_{P}(s)$ is the Young conjugate function to $\Psi_{P}(s)$, and

$$
\Phi_{P}(s)=\int_{0}^{s} r^{P^{\prime}-1}\left(\mathcal{A}_{P}^{-1}\left(r^{P^{\prime}}\right)\right)^{P^{\prime}} d r
$$


where $\mathcal{B}_{P}^{-1}(s)$ and $\mathcal{A}_{P}^{-1}(s)$ are inverses to

$$
\mathcal{B}_{P}(s)=\int_{0}^{s} \frac{\Psi(t)}{t^{1+P^{\prime}}} d t \quad \text { and } \quad \mathcal{A}_{P}(s)=\int_{0}^{s} \frac{\widetilde{\Phi}(t)}{t^{1+P^{\prime}}} d t
$$

respectively. These functions $\Psi_{P}(s)$ and $\Phi_{P}(s)$ are used below with $P=\frac{n}{\alpha}$.

Theorem 3.1 ([8]) Let $\Phi$ and $\Psi$ Young functions and $0<\alpha<n$. Then the Riesz potential $I_{\alpha}$ is bounded from $L^{\Phi}\left(\mathbb{R}^{n}\right)$ to $L^{\Psi}\left(\mathbb{R}^{n}\right)$ if and only if

$$
\int_{0}^{1} \widetilde{\Phi}(t) / t^{1+n /(n-\alpha)} d t<\infty, \quad \int_{0}^{1} \Psi(t) / t^{1+n /(n-\alpha)} d t<\infty
$$

and

$\Phi$ dominates $\Psi_{n / \alpha}$ globally and $\Phi_{n / \alpha}$ dominates $\Psi$ globally.

Lemma 3.2 ([19]) Let $\Phi$ and $\Psi$ Young functions and $\Phi_{P}, P \in(1, \infty]$, Young function defined as in (3.1). If $\int_{0}^{1} \widetilde{\Phi}(t) / t^{1+P^{\prime}} d t<\infty$ and $\Phi_{P}$ dominates $\Psi$ globally then

$$
\Phi^{-1}(r) \lesssim r^{\frac{1}{P}} \Psi^{-1}(r) \quad \text { for } r>0 .
$$

For proving our main results, we need the following estimate.

Lemma 3.3 If $B_{0}:=B\left(x_{0}, r_{0}\right)$, then $r_{0}^{\alpha} \leq C I_{\alpha} \chi_{B_{0}}(x)$ for every $x \in B_{0}$.

Proof If $x, y \in B_{0}$, then $|x-y| \leq\left|x-x_{0}\right|+\left|y-x_{0}\right|<2 r_{0}$. Since $0<\alpha<n$, we get $r_{0}^{\alpha-n} \leq$ $C|x-y|^{\alpha-n}$. Therefore

$$
I_{\alpha} \chi_{B_{0}}(x)=\int_{\mathbb{R}^{n}} \chi_{B_{0}}(y)|x-y|^{\alpha-n} d y=\int_{B_{0}}|x-y|^{\alpha-n} d y \geq C r_{0}^{\alpha-n}\left|B_{0}\right|=C r_{0}^{\alpha} .
$$

The following theorem is a useful necessary and sufficient result for the boundedness of $I_{\alpha}$ in the framework of generalized Orlicz-Morrey space.

Theorem 3.4 (Spanne type result) Let $\Phi, \Psi$ be Young functions and $0<\alpha<n$.

1. If the functions $(\Phi, \Psi)$ satisfy the conditions (3.2) and (3.3), then the condition

$$
\int_{t}^{\infty} \underset{r<s<\infty}{\operatorname{essinf}} \frac{\varphi_{1}(s)}{\Phi^{-1}\left(v_{n}^{-1} s^{-n}\right)} \Psi^{-1}\left(v_{n}^{-1} r^{-n}\right) \frac{d r}{r} \leq C \varphi_{2}(t)
$$

for all $t>0$, where $C>0$ does not depend on $t$, is sufficient for the boundedness of $I_{\alpha}$ from $\mathcal{M}^{\Phi, \varphi_{1}}\left(\mathbb{R}^{n}\right)$ to $\mathcal{M}^{\Psi, \varphi_{2}}\left(\mathbb{R}^{n}\right)$.

2. If the function $\varphi_{1} \in \mathcal{G}_{\Phi}$, then the condition

$$
t^{\alpha} \varphi_{1}(t) \leq C \varphi_{2}(t)
$$

for all $t>0$, where $C>0$ does not depend on $t$, is necessary for the boundedness of $I_{\alpha}$ from $\mathcal{M}^{\Phi, \varphi_{1}}\left(\mathbb{R}^{n}\right)$ to $\mathcal{M}^{\Psi, \varphi_{2}}\left(\mathbb{R}^{n}\right)$. 
3. Let the functions $(\Phi, \Psi)$ satisfy the conditions (3.2) and (3.3). If $\varphi_{1} \in \mathcal{G}_{\Phi}$ satisfies the regularity type condition

$$
\int_{t}^{\infty} \frac{\Psi^{-1}\left(v_{n}^{-1} r^{-n}\right)}{\Phi^{-1}\left(v_{n}^{-1} r^{-n}\right)} \varphi_{1}(r) \frac{d r}{r} \leq C t^{\alpha} \varphi_{1}(t)
$$

for all $t>0$, where $C>0$ does not depend on $t$, then the condition (3.5) is necessary and sufficient for the boundedness of $I_{\alpha}$ from $\mathcal{M}^{\Phi, \varphi_{1}}\left(\mathbb{R}^{n}\right)$ to $\mathcal{M}^{\Psi, \varphi_{2}}\left(\mathbb{R}^{n}\right)$.

Proof The first part of the theorem was proved in [19], Theorem 14.

We shall now prove the second part. Let $B_{0}=B\left(x_{0}, t_{0}\right)$ and $x \in B_{0}$. By Lemma 3.3 we have $t_{0}^{\alpha} \leq C I_{\alpha} \chi_{B_{0}}(x)$. Therefore, by Lemma 2.4 and Lemma 2.7

$$
\begin{aligned}
t_{0}^{\alpha} & \leq C \Psi^{-1}\left(\left|B_{0}\right|^{-1}\right)\left\|I_{\alpha} \chi_{B_{0}}\right\|_{L^{\Psi}\left(B_{0}\right)} \leq C \varphi_{2}\left(t_{0}\right)\left\|I_{\alpha} \chi_{B_{0}}\right\|_{\mathcal{M}^{\Psi, \varphi_{2}}} \\
& \leq C \varphi_{2}\left(t_{0}\right)\left\|\chi_{B_{0}}\right\|_{\mathcal{M}^{\Phi, \varphi_{1}}} \leq C \frac{\varphi_{2}\left(t_{0}\right)}{\varphi_{1}\left(t_{0}\right)} .
\end{aligned}
$$

Since this is true for every $t_{0}>0$, we are done.

The third statement of the theorem follows from the first and second parts of the theorem.

If we take $\Phi(t)=t^{p}, \Psi(t)=t^{q}, p, q \in[1, \infty)$ at Theorem 3.4 we get the following new result for generalized Morrey spaces.

Corollary 3.5 Let $0<\alpha<n$ and $p, q \in[1, \infty)$.

1. If $1<p<\frac{n}{\alpha}$ and $\frac{1}{q}=\frac{1}{p}-\frac{\alpha}{n}$, then the condition

$$
\int_{t}^{\infty} \frac{\operatorname{essinf}_{r<s<\infty} \varphi_{1}(s) s^{\frac{n}{p}}}{r^{\frac{n}{q}}} \frac{d r}{r} \leq C \varphi_{2}(t)
$$

for all $t>0$, where $C>0$ does not depend on $t$, is sufficient for the boundedness of $I_{\alpha}$ from $\mathcal{M}^{p, \varphi_{1}}\left(\mathbb{R}^{n}\right)$ to $\mathcal{M}^{q, \varphi_{2}}\left(\mathbb{R}^{n}\right)$.

2. If the function $\varphi_{1} \in \mathcal{G}_{p} \equiv \mathcal{G}_{t^{p}}$, then the condition (3.5) is necessary for the boundedness of $I_{\alpha}$ from $\mathcal{M}^{p, \varphi_{1}}\left(\mathbb{R}^{n}\right)$ to $\mathcal{M}^{q, \varphi_{2}}\left(\mathbb{R}^{n}\right)$.

3. Let $1<p<\frac{n}{\alpha}$ and $\frac{1}{q}=\frac{1}{p}-\frac{\alpha}{n}$. If $\varphi_{1} \in \mathcal{G}_{p}$ satisfies the regularity condition

$$
\int_{t}^{\infty} r^{\alpha} \varphi_{1}(r) \frac{d r}{r} \leq C t^{\alpha} \varphi_{1}(t)
$$

for all $t>0$, where $C>0$ does not depend on $t$, then the condition (3.5) is necessary and sufficient for the boundedness of $I_{\alpha}$ from $\mathcal{M}^{p, \varphi_{1}}\left(\mathbb{R}^{n}\right)$ to $\mathcal{M}^{q, \varphi_{2}}\left(\mathbb{R}^{n}\right)$.

Remark 3.6 If we take $\varphi_{1}(t)=t^{\frac{\lambda-n}{p}}$ and $\varphi_{2}(t)=t^{\frac{\mu-n}{q}}$ at Corollary 3.5, then conditions (3.8) and (3.5) are equivalent to $0<\lambda<n-\alpha p$ and $\frac{\lambda}{p}=\frac{\mu}{q}$, respectively. Therefore, we get the following Spanne result for Morrey spaces.

Corollary 3.7 ([20]) Let $0<\alpha<n, 1<p<\frac{n}{\alpha}, 0<\lambda<n-\alpha p$ and $\frac{1}{q}=\frac{1}{p}-\frac{\alpha}{n}$. Then $I_{\alpha}$ is bounded from $\mathcal{M}^{p, \lambda}\left(\mathbb{R}^{n}\right)$ to $\mathcal{M}^{q, \mu}\left(\mathbb{R}^{n}\right)$ if and only if $\frac{\lambda}{p}=\frac{\mu}{q}$. 


\section{Adams type results for $I_{\alpha}$ in the spaces $\mathcal{M}^{\Phi, \varphi}$}

The following pointwise estimate plays a key role where we prove our main results.

Lemma 4.1 Let $0<\alpha<n$ and $\varphi(x, t)$ satisfy the condition

$$
t^{\alpha} \varphi(x, t)+\int_{t}^{\infty} r^{\alpha} \varphi(x, r) \frac{d r}{r} \leq C \varphi(x, t)^{\beta}
$$

for some $\beta \in(0,1)$ and for every $x \in \mathbb{R}^{n}$ and $t>0$. Then we have the pointwise inequality:

$$
\left|I_{\alpha} f(x)\right| \lesssim(M f(x))^{\beta}\|f\|_{\mathcal{M}^{\Phi, \varphi}}^{1-\beta}
$$

Proof For an arbitrary ball $B=B(x, t)$ we represent $f$ as

$$
f=f_{1}+f_{2}, \quad f_{1}(y)=f(y) \chi_{B}(y), \quad f_{2}(y)=f(y) \chi_{\mathrm{c}_{B}}(y)
$$

and have

$$
I_{\alpha} f(x)=I_{\alpha} f_{1}(x)+I_{\alpha} f_{2}(x)
$$

For $I_{\alpha} f_{1}(x)$, following the Hedberg trick, see [21], we obtain $\left|I_{\alpha} f_{1}(x)\right| \leq C_{1} t^{\alpha} M f(x)$. For $I_{\alpha} f_{2}(x)$ by Lemma 2.3 we have

$$
\begin{aligned}
\int_{\mathrm{C}_{(B(x, t))}} \frac{|f(y)|}{|x-y|^{n-\alpha}} d y & \approx \int_{\mathrm{C}_{(B(x, t))}}|f(y)| \int_{|x-y|}^{\infty} \frac{d r}{r^{n+1-\alpha}} d y \\
& \approx \int_{t}^{\infty} \int_{t \leq|x-y|<r}|f(y)| d y \frac{d r}{r^{n+1-\alpha}} \\
& \leq C_{2} \int_{t}^{\infty} \Phi^{-1}\left(|B(x, r)|^{-1}\right) r^{\alpha-1}\|f\|_{L^{\Phi}(B(x, r))} d r .
\end{aligned}
$$

Consequently we have

$$
\begin{aligned}
\left|I_{\alpha} f(x)\right| & \lesssim t^{\alpha} M f(x)+\int_{t}^{\infty} \Phi^{-1}\left(|B(x, r)|^{-1}\right) r^{\alpha-1}\|f\|_{L^{\Phi}(B(x, r))} d r \\
& \lesssim t^{\alpha} M f(x)+\|f\|_{\mathcal{M}^{\Phi, \varphi}} \int_{t}^{\infty} r^{\alpha} \varphi(x, r) \frac{d r}{r} .
\end{aligned}
$$

Thus, by (4.1) we obtain

$$
\begin{aligned}
\left|I_{\alpha} f(x)\right| & \lesssim \min \left\{\varphi(x, t)^{\beta-1} M f(x), \varphi(x, t)^{\beta}\|f\|_{\mathcal{M}^{\Phi, \varphi}}\right\} \\
& \lesssim \sup _{s>0} \min \left\{s^{\beta-1} M f(x), s^{\beta}\|f\|_{\mathcal{M}^{\Phi, \varphi}}\right\} \\
& =(M f(x))^{\beta}\|f\|_{\mathcal{M}^{\Phi, \varphi}}^{1-\beta},
\end{aligned}
$$

where we have used the fact that the supremum is achieved when the minimum parts are balanced. 
Theorem 4.2 Let $\Phi \in \mathcal{Y} \cap \nabla_{2}$ and $0<\alpha<n$. Let $\varphi(x, t)$ satisfy the conditions (2.3) and (4.1). Define $\eta(x, t) \equiv \varphi(x, t)^{\beta}$ and $\Psi(t) \equiv \Phi\left(t^{1 / \beta}\right)$. Then the operator $I_{\alpha}$ is bounded from $\mathcal{M}^{\Phi, \varphi}\left(\mathbb{R}^{n}\right)$ to $\mathcal{M}^{\Psi, \eta}\left(\mathbb{R}^{n}\right)$.

Proof By using the pointwise estimate (4.2) we have

$$
\left\|I_{\alpha} f\right\|_{L^{\Psi}(B)} \lesssim\left\|(M f)^{\beta}\right\|_{L^{\Psi}(B)}\|f\|_{\mathcal{M}^{\Phi, \varphi}}^{1-\beta}
$$

where $B=B(x, t)$.

Note that from (2.2) we get

$$
\int_{B} \Psi\left(\frac{(M f(x))^{\beta}}{\|M f\|_{L^{\Phi}(B)}^{\beta}}\right) d x=\int_{B} \Phi\left(\frac{M f(x)}{\|M f\|_{L^{\Phi}(B)}}\right) d x \leq 1 .
$$

Thus $\left\|(M f)^{\beta}\right\|_{L^{\Psi}(B)} \leq\|M f\|_{L^{\Phi}(B)^{\prime}}^{\beta}$. Consequently by using this inequality we have

$$
\left\|I_{\alpha} f\right\|_{L^{\Psi}(B)} \lesssim\|M f\|_{L^{\Phi}(B)}^{\beta}\|f\|_{\mathcal{M}^{\Phi, \varphi}}^{1-\beta} .
$$

From Theorem 2.6 and (4.3), we get

$$
\begin{aligned}
\left\|I_{\alpha} f\right\|_{\mathcal{M}^{\Psi, \eta}} & =\sup _{x \in \mathbb{R}^{n}, t>0} \eta(x, t)^{-1} \Psi^{-1}\left(|B|^{-1}\right)\left\|I_{\alpha} f\right\|_{L^{\Psi}(B)} \\
& \lesssim\|f\|_{\mathcal{M}^{\Phi, \varphi}}^{1-\beta} \sup _{x \in \mathbb{R}^{n}, t>0} \eta(x, t)^{-1} \Psi^{-1}\left(|B|^{-1}\right)\|M f\|_{L^{\Phi}(B)}^{\beta} \\
& =\|f\|_{\mathcal{M}^{\Phi, \varphi}}^{1-\beta}\left(\sup _{x \in \mathbb{R}^{n}, t>0} \varphi(x, t)^{-1} \Phi^{-1}\left(|B|^{-1}\right)\|M f\|_{L^{\Phi}(B)}\right)^{\beta} \\
& \lesssim\|f\|_{\mathcal{M}^{\Phi, \varphi}} .
\end{aligned}
$$

The following theorem is one of our main results.

Theorem 4.3 (Adams type result) Let $0<\alpha<n, \Phi \in \mathcal{Y}, \beta \in(0,1)$ and $\eta(t) \equiv \varphi(t)^{\beta}$ and $\Psi(t) \equiv \Phi\left(t^{1 / \beta}\right)$.

1. If $\Phi \in \nabla_{2}$ and $\varphi(t)$ satisfies (2.3), then the condition

$$
t^{\alpha} \varphi(t)+\int_{t}^{\infty} r^{\alpha} \varphi(r) \frac{d r}{r} \leq C \varphi(t)^{\beta}
$$

for all $t>0$, where $C>0$ does not depend on $t$, is sufficient for the boundedness of $I_{\alpha}$ from $\mathcal{M}^{\Phi, \varphi}\left(\mathbb{R}^{n}\right)$ to $\mathcal{M}^{\Psi, \eta}\left(\mathbb{R}^{n}\right)$.

2. If $\varphi \in \mathcal{G}_{\Phi}$, then the condition

$$
t^{\alpha} \varphi(t) \leq C \varphi(t)^{\beta}
$$

for all $t>0$, where $C>0$ does not depend on $t$, is necessary for the boundedness of $I_{\alpha}$ from $\mathcal{M}^{\Phi, \varphi}\left(\mathbb{R}^{n}\right)$ to $\mathcal{M}^{\Psi, \eta}\left(\mathbb{R}^{n}\right)$. 
3. Let $\Phi \in \nabla_{2}$. If $\varphi \in \mathcal{G}_{\Phi}$ satisfies the regularity condition

$$
\int_{t}^{\infty} r^{\alpha} \varphi(r) \frac{d r}{r} \leq C t^{\alpha} \varphi(t)
$$

for all $t>0$, where $C>0$ does not depend on $t$, then the condition (4.5) is necessary and sufficient for the boundedness of $I_{\alpha}$ from $\mathcal{M}^{\Phi, \varphi}\left(\mathbb{R}^{n}\right)$ to $\mathcal{M}^{\Psi, \eta}\left(\mathbb{R}^{n}\right)$.

Proof The first part of the theorem is a corollary of Theorem 4.2.

We shall now prove the second part. Let $B_{0}=B\left(x_{0}, t_{0}\right)$ and $x \in B_{0}$. By Lemma 3.3 we have $t_{0}^{\alpha} \leq C I_{\alpha} \chi_{B_{0}}(x)$. Therefore, by Lemma 2.4 and Lemma 2.7 we have

$$
\begin{aligned}
t_{0}^{\alpha} & \leq C \Psi^{-1}\left(\left|B_{0}\right|^{-1}\right)\left\|I_{\alpha} \chi_{B_{0}}\right\|_{L^{\Psi}\left(B_{0}\right)} \leq C \eta\left(t_{0}\right)\left\|I_{\alpha} \chi_{B_{0}}\right\|_{\mathcal{M}^{\Psi, \eta}} \\
& \leq C \eta\left(t_{0}\right)\left\|\chi_{B_{0}}\right\|_{\mathcal{M}^{\Phi, \varphi}} \leq C \frac{\eta\left(t_{0}\right)}{\varphi\left(t_{0}\right)}=C \varphi\left(t_{0}\right)^{\beta-1} .
\end{aligned}
$$

Since this is true for every $t_{0}>0$, we are done.

The third statement of the theorem follows from the first and second parts of the theorem.

If we take $\Phi(t)=t^{p}, p \in[1, \infty)$ and $\beta=\frac{p}{q}$ with $p<q<\infty$ at Theorem 4.3 we get the following new result for generalized Morrey spaces.

Corollary 4.4 Let $1<p<q<\infty$.

1. If $\varphi(t)$ satisfies (1.1), then the condition

$$
t^{\alpha} \varphi(t)+\int_{t}^{\infty} r^{\alpha} \varphi(r) \frac{d r}{r} \leq C \varphi(t)^{\frac{p}{q}}
$$

for all $t>0$, where $C>0$ does not depend on $t$, is sufficient for the boundedness of $I_{\alpha}$ from $\mathcal{M}^{p, \varphi}\left(\mathbb{R}^{n}\right)$ to $\mathcal{M}^{q, \varphi^{\frac{p}{q}}}\left(\mathbb{R}^{n}\right)$.

2. If $\varphi \in \mathcal{G}_{p}$, then the condition

$$
t^{\alpha} \varphi(t) \leq C \varphi(t)^{\frac{p}{q}}
$$

for all $t>0$, where $C>0$ does not depend on $t$, is necessary for the boundedness of $I_{\alpha}$ from $\mathcal{M}^{p, \varphi}\left(\mathbb{R}^{n}\right)$ to $\mathcal{M}^{q, \varphi} \frac{p}{q}\left(\mathbb{R}^{n}\right)$.

3. If $\varphi \in \mathcal{G}_{p}$ satisfies the regularity condition (4.6), then the condition (4.8) is necessary and sufficient for the boundedness of $I_{\alpha}$ from $\mathcal{M}^{p, \varphi}\left(\mathbb{R}^{n}\right)$ to $\mathcal{M}^{q, \varphi^{\frac{p}{q}}}\left(\mathbb{R}^{n}\right)$.

Remark 4.5 If we take $\varphi(t)=t^{\frac{\lambda-n}{p}}$ at Corollary 4.4, then the condition (4.6) is equivalent to $0<\lambda<n-\alpha p$ and the condition (4.8) is equivalent to $\frac{1}{p}-\frac{1}{q}=\frac{\alpha}{n-\lambda}$. Therefore, we get the following Adams result for Morrey spaces.

Corollary 4.6 Let $0<\alpha<n, 1<p<q<\infty$ and $0<\lambda<n-\alpha p$. Then $I_{\alpha}$ is bounded from $\mathcal{M}^{p, \lambda}\left(\mathbb{R}^{n}\right)$ to $\mathcal{M}^{q, \lambda}\left(\mathbb{R}^{n}\right)$ if and only if $\frac{1}{p}-\frac{1}{q}=\frac{\alpha}{n-\lambda}$. 


\section{Spanne type results for $\left[b, l_{\alpha}\right]$ in the spaces $\mathcal{M}^{\Phi, \varphi}$}

We recall the definition of the space of $\operatorname{BMO}\left(\mathbb{R}^{n}\right)$.

Definition 5.1 Suppose that $f \in L_{\text {loc }}^{1}\left(\mathbb{R}^{n}\right)$, let

$$
\|f\|_{*}=\sup _{x \in \mathbb{R}^{n}, r>0} \frac{1}{|B(x, r)|} \int_{B(x, r)}\left|f(y)-f_{B(x, r)}\right| d y<\infty,
$$

where

$$
f_{B(x, r)}=\frac{1}{|B(x, r)|} \int_{B(x, r)} f(y) d y .
$$

Define

$$
\operatorname{BMO}\left(\mathbb{R}^{n}\right)=\left\{f \in L_{\mathrm{loc}}^{1}\left(\mathbb{R}^{n}\right):\|f\|_{*}<\infty\right\} .
$$

Modulo constants, the space $\mathrm{BMO}\left(\mathbb{R}^{n}\right)$ is a Banach space with respect to the norm $\|\cdot\|_{*}$. Before proving our theorems, we need the following lemmas.

Lemma $5.2([22])$ Let $b \in \mathrm{BMO}\left(\mathbb{R}^{n}\right)$. Then there is a constant $C>0$ such that

$$
\left|b_{B(x, r)}-b_{B(x, t)}\right| \leq C\|b\|_{*} \ln \frac{t}{r} \quad \text { for } 0<2 r<t
$$

where $C$ is independent of $b, x, r$, and $t$.

Lemma 5.3 $([19,23])$ Let $f \in \mathrm{BMO}\left(\mathbb{R}^{n}\right)$ and $\Phi$ be a Young function with $\Phi \in \Delta_{2}$, then

$$
\|f\|_{*} \approx \sup _{x \in \mathbb{R}^{n}, r>0} \Phi^{-1}\left(|B(x, r)|^{-1}\right)\left\|f(\cdot)-f_{B(x, r)}\right\|_{L^{\Phi}(B(x, r))^{\circ}}
$$

Lemma 5.4 If $b \in L_{\mathrm{loc}}^{1}\left(\mathbb{R}^{n}\right)$ and $B_{0}:=B\left(x_{0}, r_{0}\right)$, then

$$
r_{0}^{\alpha}\left|b(x)-b_{B_{0}}\right| \leq C\left|b, I_{\alpha}\right| \chi_{B_{0}}(x)
$$

for every $x \in B_{0}$, where $b_{B_{0}}=\frac{1}{\left|B_{0}\right|} \int_{B_{0}} b(y) d y$.

Proof If $x, y \in B_{0}$, then $|x-y| \leq\left|x-x_{0}\right|+\left|y-x_{0}\right|<2 r_{0}$. Since $0<\alpha<n$, we get $r_{0}^{\alpha-n} \leq$ $C|x-y|^{\alpha-n}$. Therefore

$$
\begin{aligned}
\left|b, I_{\alpha}\right| \chi_{B_{0}}(x) & =\int_{B_{0}}|b(x)-b(y)||x-y|^{\alpha-n} d y \geq C r_{0}^{\alpha-n} \int_{B_{0}}|b(x)-b(y)| d y \\
& \geq C r_{0}^{\alpha-n}\left|\int_{B_{0}}(b(x)-b(y)) d y\right|=C r_{0}^{\alpha}\left|b(x)-b_{B_{0}}\right| .
\end{aligned}
$$

The following theorem is one of our main results.

Theorem 5.5 Let $0<\alpha<n$ and $b \in \mathrm{BMO}\left(\mathbb{R}^{n}\right)$. 
1. Let $\Phi$ be a Youngfunction and $\Psi$ defined, via its inverse, by setting, for all $t \in(0, \infty)$, $\Psi^{-1}(t):=\Phi^{-1}(t) t^{-\alpha / n}$. If $\Phi, \Psi \in \Delta_{2} \cap \nabla_{2}$, then the condition

$$
\int_{r}^{\infty}\left(1+\ln \frac{t}{r}\right) \underset{t<s<\infty}{\operatorname{sins}} \frac{\varphi_{1}(s)}{\Phi^{-1}\left(s^{-n}\right)} \Psi^{-1}\left(t^{-n}\right) \frac{d t}{t} \leq C \varphi_{2}(r)
$$

for all $r>0$, where $C>0$ does not depend on $r$, is sufficient for the boundedness of $\left|b, I_{\alpha}\right|$ from $\mathcal{M}^{\Phi, \varphi_{1}}\left(\mathbb{R}^{n}\right)$ to $\mathcal{M}^{\Psi, \varphi_{2}}\left(\mathbb{R}^{n}\right)$.

2. Let $\Phi, \Psi$ be Young functions. If $\Psi \in \Delta_{2}$ and $\varphi_{1} \in \mathcal{G}_{\Phi}$, then the condition (3.5) is necessary for the boundedness of $\left|b, I_{\alpha}\right|$ from $\mathcal{M}^{\Phi, \varphi_{1}}\left(\mathbb{R}^{n}\right)$ to $\mathcal{M}^{\Psi, \varphi_{2}}\left(\mathbb{R}^{n}\right)$.

3. Let $\Phi$ be a Young function and $\Psi$ defined, via its inverse, by setting, for all $t \in(0, \infty)$, $\Psi^{-1}(t):=\Phi^{-1}(t) t^{-\alpha / n}$ and $\Phi, \Psi \in \Delta_{2} \cap \nabla_{2}$. If $\varphi_{1} \in \mathcal{G}_{\Phi}$ satisfies the regularity type condition

$$
\int_{r}^{\infty}\left(1+\ln \frac{t}{r}\right) t^{\alpha} \varphi_{1}(t) \frac{d t}{t} \leq C^{\alpha} \varphi_{1}(r)
$$

for all $r>0$, where $C>0$ does not depend on $r$, then the condition (3.5) is necessary and sufficient for the boundedness of $\left|b, I_{\alpha}\right|$ from $\mathcal{M}^{\Phi, \varphi_{1}}\left(\mathbb{R}^{n}\right)$ to $\mathcal{M}^{\Psi, \varphi_{2}}\left(\mathbb{R}^{n}\right)$.

Proof From the proof of [19], Theorem 34, we know that the boundedness result is still true if one has $\left|b, I_{\alpha}\right|$ instead of $\left[b, I_{\alpha}\right]$. Hence, the first part of the theorem is a corollary of [19], Theorem 34.

We shall now prove the second part. Let $B_{0}=B\left(x_{0}, r_{0}\right)$ and $x \in B_{0}$. By Lemma 5.4 we have $r_{0}^{\alpha}\left|b(x)-b_{B_{0}}\right| \leq C\left|b, I_{\alpha}\right| \chi_{B_{0}}(x)$. Therefore, by Lemma 5.3 and Lemma 2.7

$$
\begin{aligned}
r_{0}^{\alpha} & \leq C \frac{\left\|b, I_{\alpha} \mid \chi_{B_{0}}\right\|_{L^{\Psi}\left(B_{0}\right)}}{\left\|b(\cdot)-b_{B_{0}}\right\|_{L^{\Psi}\left(B_{0}\right)}} \leq \frac{C}{\|b\|_{*}}\left\|\left|b, I_{\alpha}\right| \chi_{B_{0}}\right\|_{L^{\Psi}\left(B_{0}\right)} \Psi^{-1}\left(\left|B_{0}\right|^{-1}\right) \\
& \leq \frac{C}{\|b\|_{*}} \varphi_{2}\left(r_{0}\right)\left\|\left|b, I_{\alpha}\right| \chi_{B_{0}}\right\|_{\mathcal{M}^{\Psi, \varphi_{2}}} \leq C \varphi_{2}\left(r_{0}\right)\left\|\chi_{B_{0}}\right\|_{\mathcal{M}^{\Phi, \varphi_{1}}} \leq C \frac{\varphi_{2}\left(r_{0}\right)}{\varphi_{1}\left(r_{0}\right)} .
\end{aligned}
$$

Since this is true for every $r_{0}>0$, we are done.

The third statement of the theorem follows from the first and second parts of the theorem.

If we take $\Phi(t)=t^{p}, \Psi(t)=t^{q}, p, q \in[1, \infty)$ at Theorem 5.5 we get the following new result for generalized Morrey spaces.

Corollary 5.6 Let $0<\alpha<n, p, q \in[1, \infty)$ and $b \in \mathrm{BMO}\left(\mathbb{R}^{n}\right)$.

1. Let $1<p<\frac{n}{\alpha}, \frac{1}{q}=\frac{1}{p}-\frac{\alpha}{n}$, then the condition

$$
\int_{r}^{\infty}\left(1+\ln \frac{t}{r}\right) \frac{\operatorname{essinf}_{t<s<\infty} \varphi_{1}(s) s^{\frac{n}{p}}}{t^{\frac{n}{q}+1}} d t \leq C \varphi_{2}(r)
$$

for all $r>0$, where $C>0$ does not depend on $r$, is sufficient for the boundedness of $\left|b, I_{\alpha}\right|$ from $\mathcal{M}^{p, \varphi_{1}}\left(\mathbb{R}^{n}\right)$ to $\mathcal{M}^{q, \varphi_{2}}\left(\mathbb{R}^{n}\right)$.

2. If $\varphi_{1} \in \mathcal{G}_{p}$, then the condition (3.5) is necessary for the boundedness of $\left|b, I_{\alpha}\right|$ from $\mathcal{M}^{p, \varphi_{1}}\left(\mathbb{R}^{n}\right)$ to $\mathcal{M}^{q, \varphi_{2}}\left(\mathbb{R}^{n}\right)$. 
3. Let $1<p<\frac{n}{\alpha}, \frac{1}{q}=\frac{1}{p}-\frac{\alpha}{n}$. If $\varphi_{1} \in \mathcal{G}_{p}$ satisfies the regularity type condition (5.3), then the condition (3.5) is necessary and sufficient for the boundedness of $\left|b, I_{\alpha}\right|$ from $\mathcal{M}^{p, \varphi_{1}}\left(\mathbb{R}^{n}\right)$ to $\mathcal{M}^{q, \varphi_{2}}\left(\mathbb{R}^{n}\right)$.

\section{Adams type results for $\left[b, I_{\alpha}\right]$ in the spaces $\mathcal{M}^{\Phi, \varphi}$}

The commutators generated by $b \in L_{\text {loc }}^{1}\left(\mathbb{R}^{n}\right)$ and the maximal operator $M$ is defined by

$$
M_{b}(f)(x)=\sup _{t>0}|B(x, t)|^{-1} \int_{B(x, t)}|b(x)-b(y)||f(y)| d y .
$$

Theorem 6.1 ([24]) Let $b \in \mathrm{BMO}\left(\mathbb{R}^{n}\right)$, $\Phi$ be a Young function with $\Phi \in \Delta_{2} \cap \nabla_{2}, \varphi$ and $\Phi$ satisfy the condition

$$
\sup _{r<t<\infty}\left(1+\ln \frac{t}{r}\right) \Phi^{-1}\left(|B(x, t)|^{-1}\right) \underset{t<s<\infty}{\operatorname{essinf}} \frac{\varphi(x, s)}{\Phi^{-1}\left(|B(x, s)|^{-1}\right)} \leq C \varphi(x, r)
$$

where $C$ does not depend on $x$ and $r$. Then the operator $M_{b}$ is bounded from $\mathcal{M}^{\Phi, \varphi}\left(\mathbb{R}^{n}\right)$ to $\mathcal{M}^{\Phi, \varphi}\left(\mathbb{R}^{n}\right)$.

The following lemma is the analog of the Hedberg trick for $\left[b, I_{\alpha}\right]$.

Lemma 6.2 If $0<\alpha<n$ and $f, b \in L_{\mathrm{loc}}^{1}\left(\mathbb{R}^{n}\right)$, then for all $x \in \mathbb{R}^{n}$ and $r>0$ we get

$$
\int_{B(x, r)} \frac{|f(y)|}{|x-y|^{n-\alpha}}|b(x)-b(y)| d y \lesssim r^{\alpha} M_{b} f(x)
$$

Proof

$$
\begin{aligned}
& \int_{B(x, r)} \frac{|f(y)|}{|x-y|^{n-\alpha}}|b(x)-b(y)| d y \\
& \quad=\sum_{j=0}^{\infty} \int_{2^{-j-1} r \leq|x-y|<2^{-j_{r}}} \frac{|f(y)|}{|x-y|^{n-\alpha}}|b(x)-b(y)| d y \\
& \quad \lesssim \sum_{j=0}^{\infty}\left(2^{-j} r\right)^{\alpha}\left(2^{-j} r\right)^{-n} \int_{|x-y|<2^{-j} r_{r}}|f(y)||b(x)-b(y)| d y \\
& \quad \lesssim r^{\alpha} M_{b} f(x) .
\end{aligned}
$$

Theorem 6.3 Let $b \in \operatorname{BMO}\left(\mathbb{R}^{n}\right), \beta \in(0,1)$, $\Phi$ be a Young function with $\Phi \in \Delta_{2} \cap \nabla_{2}$. Let $\varphi(x, r)$ satisfy the conditions (6.1) and

$$
r^{\alpha} \varphi(x, r)+\int_{r}^{\infty}\left(1+\ln \frac{t}{r}\right) \varphi(x, t) t^{\alpha} \frac{d t}{t} \leq C \varphi(x, r)^{\beta}
$$

for every $x \in \mathbb{R}^{n}$ and $r>0$. Define $\eta(x, r) \equiv \varphi(x, r)^{\beta}$ and $\Psi(r) \equiv \Phi\left(r^{1 / \beta}\right)$. Then the operator $\left[b, I_{\alpha}\right]$ is bounded from $\mathcal{M}^{\Phi, \varphi}\left(\mathbb{R}^{n}\right)$ to $\mathcal{M}^{\Psi, \eta}\left(\mathbb{R}^{n}\right)$.

Proof For arbitrary $x_{0} \in \mathbb{R}^{n}$, set $B=B\left(x_{0}, r\right)$ for the ball centered at $x_{0}$ and of radius $r$. Write $f=f_{1}+f_{2}$ with $f_{1}=f \chi_{2 B}$ and $f_{2}=f \chi_{\left.\mathrm{c}_{(2 B)}\right)}$. 
For $x \in B$ we have

$$
\begin{aligned}
\left|\left[b, I_{\alpha}\right] f_{2}(x)\right| & \lesssim \int_{\mathbb{R}^{n}} \frac{|b(y)-b(x)|}{|x-y|^{n-\alpha}}\left|f_{2}(y)\right| d y \approx \int_{\mathrm{C}_{(2 B)}} \frac{|b(y)-b(x)|}{\left|x_{0}-y\right|^{n-\alpha}}|f(y)| d y \\
& \lesssim \int_{\mathrm{C}_{(2 B)}} \frac{\left|b(y)-b_{B}\right|}{\left|x_{0}-y\right|^{n-\alpha}}|f(y)| d y+\int_{\mathrm{C}_{(2 B)}} \frac{\left|b(x)-b_{B}\right|}{\left|x_{0}-y\right|^{n-\alpha}}|f(y)| d y=J_{1}+J_{2}(x),
\end{aligned}
$$

since $x \in B$ and $y \in{ }^{\mathrm{C}}(2 B)$ implies $|x-y| \approx\left|x_{0}-y\right|$.

Let us estimate $J_{1}$ :

$$
\begin{aligned}
J_{1} & =\int_{\mathrm{C}_{(2 B)}} \frac{\left|b(y)-b_{B}\right|}{\left|x_{0}-y\right|^{n-\alpha}}|f(y)| d y \approx \int_{\mathrm{C}_{(2 B)}}\left|b(y)-b_{B}\right||f(y)| \int_{\left|x_{0}-y\right|}^{\infty} \frac{d t}{t^{n+1-\alpha}} d y \\
& \approx \int_{2 r}^{\infty} \int_{2 r \leq\left|x_{0}-y\right| \leq t}\left|b(y)-b_{B}\right||f(y)| d y \frac{d t}{t^{n+1-\alpha}} \lesssim \int_{2 r}^{\infty} \int_{B\left(x_{0}, t\right)}\left|b(y)-b_{B}\right||f(y)| d y \frac{d t}{t^{n+1-\alpha}}
\end{aligned}
$$

Applying Hölder's inequality, by (2.1), (5.1), (5.2), and Lemma 2.3 we get

$$
\begin{aligned}
J_{1} \lesssim & \int_{2 r}^{\infty} \int_{B\left(x_{0}, t\right)}\left|b(y)-b_{B\left(x_{0}, t\right)}\right| f(y) \mid d y \frac{d t}{t^{n+1-\alpha}} \\
& +\int_{2 r}^{\infty}\left|b_{B\left(x_{0}, r\right)}-b_{B\left(x_{0}, t\right)}\right| \int_{B\left(x_{0}, t\right)}|f(y)| d y \frac{d t}{t^{n+1-\alpha}} \\
\lesssim & \int_{2 r}^{\infty}\left\|b(\cdot)-b_{B\left(x_{0}, t\right)}\right\|_{L^{\widetilde{\Phi}}\left(B\left(x_{0}, t\right)\right)}\|f\|_{L_{\Phi}\left(B\left(x_{0}, t\right)\right)} \frac{d t}{t^{n+1-\alpha}} \\
& +\int_{2 r}^{\infty}\left|b_{B\left(x_{0}, r\right)}-b_{B\left(x_{0}, t\right) \mid}\right|\|f\|_{L_{\Phi}\left(B\left(x_{0}, t\right)\right)} \Phi^{-1}\left(\left|B\left(x_{0}, t\right)\right|^{-1}\right) \frac{d t}{t^{1-\alpha}} \\
\lesssim & \|b\|_{*} \int_{2 r}^{\infty}\left(1+\ln \frac{t}{r}\right)\|f\|_{L_{\Phi}\left(B\left(x_{0}, t\right)\right)} \Phi^{-1}\left(\left|B\left(x_{0}, t\right)\right|^{-1}\right) \frac{d t}{t^{1-\alpha}} . \\
\lesssim & \|b\|_{*}\|f\|_{\mathcal{M}^{\Phi, \varphi}} \int_{2 r}^{\infty}\left(1+\ln \frac{t}{r}\right) \varphi\left(x_{0}, t\right) \frac{d t}{t^{1-\alpha}} .
\end{aligned}
$$

Also using (6.2), we get

$$
J_{0}(x)+J_{1} \lesssim\|b\|_{*} r^{\alpha} M_{b} f(x)+\|b\|_{*}\|f\|_{\mathcal{M}^{\Phi, \varphi}} \int_{2 r}^{\infty}\left(1+\ln \frac{t}{r}\right) \varphi\left(x_{0}, t\right) \frac{d t}{t^{1-\alpha}}
$$

where $J_{0}(x):=\left|\left[b, I_{\alpha}\right] f_{1}(x)\right|$.

Thus, by (6.3) we obtain

$$
\begin{aligned}
J_{0}(x)+J_{1} & \lesssim\|b\|_{*} \min \left\{\varphi\left(x_{0}, r\right)^{\beta-1} M_{b} f(x), \varphi\left(x_{0}, r\right)^{\beta}\|f\|_{\mathcal{M}^{\Phi, \varphi}}\right\} \\
& \lesssim\|b\|_{*} \sup _{s>0} \min \left\{s^{\beta-1} M_{b} f(x), s^{\beta}\|f\|_{\mathcal{M}^{\Phi, \varphi}}\right\} \\
& =\|b\|_{*}\left(M_{b} f(x)\right)^{\beta}\|f\|_{\mathcal{M}^{\Phi, \varphi}}^{1-\beta} .
\end{aligned}
$$

Consequently for every $x \in B$ we have

$$
J_{0}(x)+J_{1} \lesssim\|b\|_{*}\left(M_{b} f(x)\right)^{\beta}\|f\|_{\mathcal{M}^{\Phi, \varphi}}^{1-\beta} .
$$


By using the inequality (6.4) we have

$$
\left\|J_{0}(\cdot)+J_{1}\right\|_{L^{\Psi}(B)} \lesssim\|b\|_{*}\left\|\left(M_{b} f\right)^{\beta}\right\|_{L^{\Psi}(B)}\|f\|_{\mathcal{M}^{\Phi, \varphi}}^{1-\beta} .
$$

Note that from (2.2) we get

$$
\int_{B} \Psi\left(\frac{\left(M_{b} f(x)\right)^{\beta}}{\left\|M_{b} f\right\|_{L^{\Phi}(B)}^{\beta}}\right) d x=\int_{B} \Phi\left(\frac{M_{b} f(x)}{\left\|M_{b} f\right\|_{L^{\Phi}(B)}}\right) d x \leq 1 .
$$

Thus $\left\|\left(M_{b} f\right)^{\beta}\right\|_{L^{\Psi}(B)} \leq\left\|M_{b} f\right\|_{L^{\Phi}(B)}^{\beta}$. Therefore, we have

$$
\left\|J_{0}(\cdot)+J_{1}\right\|_{L^{\Psi}(B)} \lesssim\|b\|_{*}\left\|M_{b} f\right\|_{L^{\Phi}(B)}^{\beta}\|f\|_{\mathcal{M}^{\Phi, \varphi}}^{1-\beta} .
$$

In order to estimate $J_{2}$, by (5.2), Lemma 2.3, and the condition (6.3), we also get

$$
\begin{aligned}
\left\|J_{2}\right\|_{L^{\Psi}(B)} & =\left\|\int_{\mathrm{c}_{(2 B)}} \frac{\left|b(\cdot)-b_{B}\right|}{\left|x_{0}-y\right|^{n-\alpha}}|f(y)| d y\right\|_{L^{\Psi}(B)} \\
& \approx\left\|b(\cdot)-b_{B}\right\|_{L^{\Psi}(B)} \int_{\mathrm{c}_{(2 B)}} \frac{|f(y)|}{\left|x_{0}-y\right|^{n-\alpha}} d y \\
& \lesssim\|b\|_{*} \frac{1}{\Psi^{-1}\left(|B|^{-1}\right)} \int_{\mathrm{c}_{(2 B)}} \frac{|f(y)|}{\left|x_{0}-y\right|^{n-\alpha}} d y \\
& \approx\|b\|_{*} \frac{1}{\Psi^{-1}\left(|B|^{-1}\right)} \int_{\mathrm{c}_{(2 B)}}|f(y)| \int_{\left|x_{0}-y\right|}^{\infty} \frac{d t}{t^{n+1-\alpha}} d y \\
& \approx\|b\|_{*} \frac{1}{\Psi^{-1}\left(|B|^{-1}\right)} \int_{2 r}^{\infty} \int_{2 r \leq\left|x_{0}-y\right|<t}|f(y)| d y \frac{d t}{t^{n+1-\alpha}} \\
& \lesssim\|b\|_{*} \frac{1}{\Psi^{-1}\left(|B|^{-1}\right)} \int_{2 r}^{\infty} \int_{B\left(x_{0}, t\right)}|f(y)| d y \frac{d t}{t^{n+1-\alpha}} \\
& \lesssim\|b\|_{*} \frac{1}{\Psi^{-1}\left(|B|^{-1}\right)} \int_{2 r}^{\infty}\|f\|_{L^{\Phi}\left(B\left(x_{0}, t\right)\right.} \Phi^{-1}\left(\left|B\left(x_{0}, t\right)\right|^{-1}\right) t^{\alpha-1} d t \\
& \lesssim\|b\|_{*} \frac{1}{\Psi^{-1}\left(|B|^{-1}\right)}\|f\|_{\mathcal{M}^{\Phi, \varphi}} \int_{2 r}^{\infty} t^{\alpha} \varphi\left(x_{0}, t\right) \frac{d t}{t} \\
& \lesssim\|b\|_{*} \frac{1}{\Psi^{-1}\left(|B|^{-1}\right)}\|f\|_{\mathcal{M}^{\Phi, \varphi} \varphi\left(x_{0}, r\right)^{\beta} .}
\end{aligned}
$$

Consequently by using Theorem 6.1, we get

$$
\begin{aligned}
\left\|\left[b, I_{\alpha}\right] f\right\|_{\mathcal{M}^{\Psi, \eta}} & =\sup _{x_{0} \in \mathbb{R}^{n}, r>0} \eta\left(x_{0}, r\right)^{-1} \Psi^{-1}\left(|B|^{-1}\right)\left\|\left[b, I_{\alpha}\right] f\right\|_{L^{\Psi}(B)} \\
\lesssim & \|b\|_{*}\|f\|_{\mathcal{M}^{\Phi, \varphi}}^{1-\beta}\left(\sup _{x_{0} \in \mathbb{R}^{n}, r>0} \varphi\left(x_{0}, r\right)^{-1} \Phi^{-1}\left(|B|^{-1}\right)\left\|M_{b} f\right\|_{L^{\Phi}(B)}\right)^{\beta} \\
& +\|b\|_{*}\|f\|_{\mathcal{M}^{\Phi, \varphi}} \\
\lesssim & \|b\|_{*}\|f\|_{\mathcal{M}^{\Phi, \varphi}} .
\end{aligned}
$$

The following theorem is one of our main results. 
Theorem 6.4 Let $0<\alpha<n, \Phi \in \mathcal{Y}, b \in \mathrm{BMO}\left(\mathbb{R}^{n}\right), \beta \in(0,1)$ and $\eta(t) \equiv \varphi(t)^{\beta}$ and $\Psi(t) \equiv$ $\Phi\left(t^{1 / \beta}\right)$.

1. If $\Phi \in \Delta_{2} \cap \nabla_{2}$ and $\varphi(t)$ satisfies (6.1), then the condition

$$
r^{\alpha} \varphi(r)+\int_{r}^{\infty}\left(1+\ln \frac{t}{r}\right) \varphi(t) t^{\alpha} \frac{d t}{t} \leq C \varphi(r)^{\beta}
$$

for all $r>0$, where $C>0$ does not depend on $r$, is sufficient for the boundedness of $\left|b, I_{\alpha}\right|$ from $\mathcal{M}^{\Phi, \varphi}\left(\mathbb{R}^{n}\right)$ to $\mathcal{M}^{\Psi, \eta}\left(\mathbb{R}^{n}\right)$.

2. If $\Phi \in \Delta_{2}$ and $\varphi \in \mathcal{G}_{\Phi}$, then the condition (4.5) is necessary for the boundedness of $\left|b, I_{\alpha}\right|$ from $\mathcal{M}^{\Phi, \varphi}\left(\mathbb{R}^{n}\right)$ to $\mathcal{M}^{\Psi, \eta}\left(\mathbb{R}^{n}\right)$.

3. Let $\Phi \in \Delta_{2} \cap \nabla_{2}$. If $\varphi \in \mathcal{G}_{\Phi}$ satisfies the conditions

$$
\sup _{r<t<\infty}\left(1+\ln \frac{t}{r}\right) \varphi(t) \leq C \varphi(r)
$$

and

$$
\int_{r}^{\infty}\left(1+\ln \frac{t}{r}\right) \varphi(t) t^{\alpha} \frac{d t}{t} \leq C r^{\alpha} \varphi(r)
$$

for all $r>0$, where $C>0$ does not depend on $r$, then the condition (4.5) is necessary and sufficient for the boundedness of $\left|b, I_{\alpha}\right|$ from $\mathcal{M}^{\Phi, \varphi}\left(\mathbb{R}^{n}\right)$ to $\mathcal{M}^{\Psi, \eta}\left(\mathbb{R}^{n}\right)$.

Proof From the proof of Theorem 6.3, we know that the boundedness result is still true if one has $\left|b, I_{\alpha}\right|$ instead of $\left[b, I_{\alpha}\right]$. Hence, the first part of the theorem is a corollary of Theorem 6.3.

We shall now prove the second part. Let $B_{0}=B\left(x_{0}, r_{0}\right)$ and $x \in B_{0}$. By Lemma 5.4 we have $r_{0}^{\alpha}\left|b(x)-b_{B_{0}}\right| \leq C\left|b, I_{\alpha}\right| \chi_{B_{0}}(x)$. Therefore, by Lemma 5.3 and Lemma 2.7

$$
\begin{aligned}
r_{0}^{\alpha} & \leq C \frac{\left\|b, I_{\alpha} \mid \chi_{B_{0}}\right\|_{L^{\Psi}\left(B_{0}\right)}}{\left\|b(\cdot)-b_{B_{0}}\right\|_{L^{\Psi}\left(B_{0}\right)}} \leq \frac{C}{\|b\|_{*}}\left\|\left|b, I_{\alpha}\right| \chi_{B_{0}}\right\|_{L^{\Psi}\left(B_{0}\right)} \Psi^{-1}\left(\left|B_{0}\right|^{-1}\right) \\
& \leq \frac{C}{\|b\|_{*}} \eta\left(r_{0}\right)\left\|\left|b, I_{\alpha}\right| \chi_{B_{0}}\right\|_{\mathcal{M}^{\Psi, \eta}} \leq C \varphi_{2}\left(r_{0}\right)\left\|\chi_{B_{0}}\right\|_{\mathcal{M}^{\Phi, \varphi}} \leq C \frac{\eta\left(r_{0}\right)}{\varphi\left(r_{0}\right)} \leq C \varphi\left(r_{0}\right)^{\beta-1} .
\end{aligned}
$$

Since this is true for every $r_{0}>0$, we are done.

The third statement of the theorem follows from the first and second parts of the theorem.

If we take $\Phi(t)=t^{p}, p \in[1, \infty)$ and $\beta=\frac{p}{q}$ with $1<p<q<\infty$ at Theorem 6.4 we get the following new result for generalized Morrey spaces.

Corollary 6.5 Let $0<\alpha<n, 1 \leq p<q<\infty$ and $b \in \mathrm{BMO}\left(\mathbb{R}^{n}\right)$.

1. If $1<p<\infty$ and $\varphi(t)$ satisfies

$$
\sup _{r<t<\infty}\left(1+\ln \frac{t}{r}\right) \frac{\operatorname{ess} \inf _{t<s<\infty} \varphi_{1}(s) s^{\frac{n}{p}}}{t^{\frac{n}{p}}} \leq C \varphi_{2}(r),
$$


then the condition

$$
r^{\alpha} \varphi(r)+\int_{r}^{\infty}\left(1+\ln \frac{t}{r}\right) \varphi(t) t^{\alpha} \frac{d t}{t} \leq C \varphi(r)^{\frac{p}{q}}
$$

for all $r>0$ and $C>0$ does not depend on $r$, is sufficient for the boundedness of $\left|b, I_{\alpha}\right|$ from $\mathcal{M}^{p, \varphi}\left(\mathbb{R}^{n}\right)$ to $\mathcal{M}^{q, \varphi^{\frac{p}{q}}}\left(\mathbb{R}^{n}\right)$.

2. If $\varphi \in \mathcal{G}_{p}$, then the condition

$$
r^{\alpha} \varphi(r) \leq C \varphi(r)^{\frac{p}{q}}
$$

for all $r>0$ and $C>0$ does not depend on $r$, is necessary for the boundedness of $\left|b, I_{\alpha}\right|$ from $\mathcal{M}^{p, \varphi}\left(\mathbb{R}^{n}\right)$ to $\mathcal{M}^{q, \varphi} \frac{\frac{p}{q}}{q}\left(\mathbb{R}^{n}\right)$.

3. Let $1<p<\infty$. If $\varphi \in \mathcal{G}_{p}$ satisfies the conditions (6.5) and (6.6), then the condition (6.7) is necessary and sufficient for the boundedness of $\left|b, I_{\alpha}\right|$ from $\mathcal{M}^{p, \varphi}\left(\mathbb{R}^{n}\right)$ to $\mathcal{M}^{q, \varphi^{\frac{p}{q}}}\left(\mathbb{R}^{n}\right)$.

\section{Weak type results}

Definition 7.1 Let $\Phi$ be a Young function. The weak Orlicz space is defined as

$$
W L^{\Phi}\left(\mathbb{R}^{n}\right):=\left\{f \in L_{\mathrm{loc}}^{1}\left(\mathbb{R}^{n}\right):\|f\|_{W L^{\Phi}}<\infty\right\},
$$

where

$$
\|f\|_{W L^{\Phi}}=\inf \left\{\lambda>0: \sup _{t>0} \Phi\left(\frac{t}{\lambda}\right) d_{f}(t) \leq 1\right\},
$$

and $d_{f}(t)=\left|\left\{x \in \mathbb{R}^{n}:|f(x)|>t\right\}\right|$.

Lemma 7.2 If $0<\|f\|_{W L^{\Phi}}<\infty$, then

$$
\sup _{t>0} \Phi\left(\frac{t}{\|f\|_{W L^{\Phi}}}\right) d_{f}(t) \leq 1
$$

Proof By the definition of $\|\cdot\|_{W L^{\Phi}}$, for all $\lambda>\|f\|_{W L^{\Phi}}$ we have $\Phi\left(\frac{t}{\lambda}\right) d_{f}(t) \leq 1$ for all $t>0$. Now as $\lambda$ decreases to $\|f\|_{W L^{\Phi}}$, the quotient $\frac{t}{\lambda}$ increases to $\frac{t}{\|f\|_{W L^{\Phi}}}$. By the left-continuity of $\Phi$, we have $\Phi\left(\frac{t}{\lambda}\right) \uparrow \Phi\left(\frac{t}{\|f\|_{W L^{\Phi}}}\right)$. Therefore we get the desired result.

By elementary calculations we have the following.

Lemma 7.3 Let $\Phi$ be a Young function and B a set in $\mathbb{R}^{n}$ with finite Lebesgue measure. Then

$$
\left\|\chi_{B}\right\|_{W L^{\Phi}}=\frac{1}{\Phi^{-1}\left(|B|^{-1}\right)} .
$$

Theorem 7.4 ([8]) Let $\Phi, \Psi$ Young functions and $0<\alpha<n$. Then the Riesz potential $I_{\alpha}$ is bounded from $L^{\Phi}\left(\mathbb{R}^{n}\right)$ to $W L^{\Psi}\left(\mathbb{R}^{n}\right)$ if and only if

$$
\int_{0}^{1} \widetilde{\Phi}(t) / t^{1+n /(n-\alpha)} d t<\infty \text { and } \Phi_{n / \alpha} \text { dominates } \Psi \text { globally. }
$$

Here, $\Phi_{n / \alpha}$ is the Young function defined as in (3.1). 
Definition 7.5 Let $\varphi(x, r)$ be a positive measurable function on $\mathbb{R}^{n} \times(0, \infty)$ and $\Phi$ any Young function. By $W \mathcal{M}^{\Phi, \varphi}\left(\mathbb{R}^{n}\right)$ we denote the weak generalized Orlicz-Morrey space of all functions $f \in W L_{\mathrm{loc}}^{\Phi}\left(\mathbb{R}^{n}\right)$ for which

$$
\|f\|_{W \mathcal{M}^{\Phi, \varphi}}=\sup _{x \in \mathbb{R}^{n}, r>0} \varphi(x, r)^{-1} \Phi^{-1}\left(|B(x, r)|^{-1}\right)\|f\|_{W L^{\Phi}(B(x, r))}<\infty,
$$

where $\|f\|_{W L^{\Phi}(B(x, r))}=\left\|f \chi_{B(x, r)}\right\|_{W L^{\Phi}}$.

Lemma 7.6 Let $B_{0}:=B\left(x_{0}, r_{0}\right)$. If $\varphi \in \mathcal{G}_{\Phi}$, then

$$
\left\|\chi_{B_{0}}\right\|_{W \mathcal{M}^{\Phi, \varphi}} \approx \frac{1}{\varphi\left(r_{0}\right)}
$$

Proof The proof is similar to the proof of Lemma 2.7 thanks to Lemma 7.3.

Theorem 7.7 ([11]) Let $\Phi \in \mathcal{Y}$, the functions $\varphi$ and $\Phi$ satisfy the condition (2.3), then the maximal operator $M$ is bounded from $\mathcal{M}^{\Phi, \varphi}\left(\mathbb{R}^{n}\right)$ to $W \mathcal{M}^{\Phi, \varphi}\left(\mathbb{R}^{n}\right)$.

Theorem 7.8 (Weak version of Spanne type result) Let $0<\alpha<n$ and $\Phi, \Psi$ are Young functions.

1. If the functions $(\Phi, \Psi)$ satisfy the condition (7.2), then the condition (3.4) is sufficient for the boundedness of $I_{\alpha}$ from $\mathcal{M}^{\Phi, \varphi_{1}}\left(\mathbb{R}^{n}\right)$ to $W \mathcal{M}^{\Psi, \varphi_{2}}\left(\mathbb{R}^{n}\right)$.

2. If the function $\varphi_{1} \in \mathcal{G}_{\Phi}$, then the condition (3.5) is necessary for the boundedness of $I_{\alpha}$ from $\mathcal{M}^{\Phi, \varphi_{1}}\left(\mathbb{R}^{n}\right)$ to $W \mathcal{M}^{\Psi, \varphi_{2}}\left(\mathbb{R}^{n}\right)$.

3. Let the functions $(\Phi, \Psi)$ satisfy the condition (7.2). If $\varphi_{1} \in \mathcal{G}_{\Phi}$ satisfies the condition (3.6), then the condition (3.5) is necessary and sufficient for the boundedness of $I_{\alpha}$ from $\mathcal{M}^{\Phi, \varphi_{1}}\left(\mathbb{R}^{n}\right)$ to $W \mathcal{M}^{\Psi, \varphi_{2}}\left(\mathbb{R}^{n}\right)$.

Proof The first part of the theorem was proved in [19], Theorem 14.

We shall now prove the second part. Let $B_{0}=B\left(x_{0}, t_{0}\right)$ and $x \in B_{0}$. By Lemma 3.3 we have $t_{0}^{\alpha} \leq C I_{\alpha} \chi_{B_{0}}(x)$. Therefore, by Lemmas 7.3 and 2.7

$$
\begin{aligned}
t_{0}^{\alpha} & \leq C \Psi^{-1}\left(\left|B_{0}\right|^{-1}\right)\left\|I_{\alpha} \chi_{B_{0}}\right\|_{W L^{\Psi}\left(B_{0}\right)} \leq C \varphi_{2}\left(t_{0}\right)\left\|I_{\alpha} \chi_{B_{0}}\right\|_{W \mathcal{M}^{\Psi, \varphi_{2}}} \\
& \leq C \varphi_{2}\left(t_{0}\right)\left\|\chi_{B_{0}}\right\|_{\mathcal{M}^{\Phi, \varphi_{1}}} \leq C \frac{\varphi_{2}\left(t_{0}\right)}{\varphi_{1}\left(t_{0}\right)}
\end{aligned}
$$

Since this is true for every $t_{0}>0$, we are done.

The third statement of the theorem follows from the first and second parts of the theorem.

If we take $\Phi(t)=t^{p}, \Psi(t)=t^{q}, p, q \in[1, \infty)$ at Theorem 7.8 we get the following new result for generalized Morrey spaces.

Corollary 7.9 Let $0<\alpha<n$ and $p, q \in[1, \infty)$.

1. If $1 \leq p<\frac{n}{\alpha}$ and $\frac{1}{q}=\frac{1}{p}-\frac{\alpha}{n}$, then the condition (3.7) is sufficient for the boundedness of $I_{\alpha}$ from $\mathcal{M}^{p, \varphi_{1}}\left(\mathbb{R}^{n}\right)$ to $W \mathcal{M}^{q, \varphi_{2}}\left(\mathbb{R}^{n}\right)$.

2. If the function $\varphi_{1} \in \mathcal{G}_{p}$, then the condition (3.5) is necessary for the boundedness of $I_{\alpha}$ from $\mathcal{M}^{p, \varphi_{1}}\left(\mathbb{R}^{n}\right)$ to $W \mathcal{M}^{q, \varphi_{2}}\left(\mathbb{R}^{n}\right)$. 
3. Let $1 \leq p<\frac{n}{\alpha}$ and $\frac{1}{q}=\frac{1}{p}-\frac{\alpha}{n}$. If $\varphi_{1} \in \mathcal{G}_{p}$ satisfies the regularity condition (3.8), then the condition (3.5) is necessary and sufficient for the boundedness of $I_{\alpha}$ from $\mathcal{M}^{p, \varphi_{1}}\left(\mathbb{R}^{n}\right)$ to $W \mathcal{M}^{q, \varphi_{2}}\left(\mathbb{R}^{n}\right)$.

Remark 7.10 If we take into account Remark 3.6 we get the following weak version of the Spanne result for Morrey spaces.

Corollary 7.11 Let $0<\alpha<n, 1 \leq p<\frac{n}{\alpha}, 0<\lambda<n-\alpha p$ and $\frac{1}{q}=\frac{1}{p}-\frac{\alpha}{n}$. Then $I_{\alpha}$ is bounded from $\mathcal{M}^{p, \lambda}\left(\mathbb{R}^{n}\right)$ to $W \mathcal{M}^{q, \mu}\left(\mathbb{R}^{n}\right)$ if and only if $\frac{\lambda}{p}=\frac{\mu}{q}$.

Theorem 7.12 Let $\Phi \in \mathcal{Y}$ and $0<\alpha<n$. Let $\varphi(x, t)$ satisfy the conditions (2.3) and (4.1). Define $\eta(x, t) \equiv \varphi(x, t)^{\beta}$ and $\Psi(t) \equiv \Phi\left(t^{1 / \beta}\right)$. Then the operator $I_{\alpha}$ is bounded from $\mathcal{M}^{\Phi, \varphi}\left(\mathbb{R}^{n}\right)$ to $W \mathcal{M}^{\Psi, \eta}\left(\mathbb{R}^{n}\right)$.

Proof By using the inequality (4.2) we have

$$
\left\|I_{\alpha} f\right\|_{W L^{\Psi}(B)} \lesssim\left\|(M f)^{\beta}\right\|_{W L^{\Psi}(B)}\|f\|_{\mathcal{M}^{\Phi, \varphi}}^{1-\beta}
$$

where $B=B(x, t)$. Note that from (7.1) we get

$$
\sup _{t>0} \Psi\left(\frac{t^{\beta}}{\|M f\|_{W L^{\Phi}(B)}^{\beta}}\right) d_{(M f)^{\beta}}\left(t^{\beta}\right)=\sup _{t>0} \Phi\left(\frac{t}{\|M f\|_{W L^{\Phi}(B)}}\right) d_{M f}(t) \leq 1 .
$$

Thus $\left\|(M f)^{\beta}\right\|_{W L^{\Psi}(B)} \leq\|M f\|_{W L^{\Phi}(B)}^{\beta}$. Consequently by using this inequality we have

$$
\left\|I_{\alpha} f\right\|_{W L^{\Psi}(B)} \lesssim\|M f\|_{W L^{\Phi}(B)}^{\beta}\|f\|_{\mathcal{M}^{\Phi, \varphi}}^{1-\beta}
$$

From Theorem 7.7 and (7.3), we get

$$
\begin{aligned}
\left\|I_{\alpha} f\right\|_{W \mathcal{M}^{\Psi, \eta}} & =\sup _{x \in \mathbb{R}^{n}, t>0} \eta(x, t)^{-1} \Psi^{-1}\left(|B|^{-1}\right)\left\|I_{\alpha} f\right\|_{W L^{\Psi}(B)} \\
& \lesssim\|f\|_{\mathcal{M}^{\Phi, \varphi}}^{1-\beta} \sup _{x \in \mathbb{R}^{n}, t>0} \eta(x, t)^{-1} \Psi^{-1}\left(|B|^{-1}\right)\|M f\|_{W L^{\Phi}(B)}^{\beta} \\
& =\|f\|_{\mathcal{M}^{\Phi, \varphi}}^{1-\beta}\left(\sup _{x \in \mathbb{R}^{n}, t>0} \varphi(x, t)^{-1} \Phi^{-1}\left(|B|^{-1}\right)\|M f\|_{W L^{\Phi}(B)}\right)^{\beta} \\
& \lesssim\|f\|_{\mathcal{M}^{\Phi, \varphi} .}
\end{aligned}
$$

Theorem 7.13 (Weak version of Adams type result) Let $0<\alpha<n, \Phi \in \mathcal{Y}, \beta \in(0,1)$ and $\eta(t) \equiv \varphi(t)^{\beta}$ and $\Psi(t) \equiv \Phi\left(t^{1 / \beta}\right)$.

1. If $\varphi(t)$ satisfies (2.3), then the condition (4.4) is sufficient for the boundedness of $I_{\alpha}$ from $\mathcal{M}^{\Phi, \varphi}\left(\mathbb{R}^{n}\right)$ to $W \mathcal{M}^{\Psi, \eta}\left(\mathbb{R}^{n}\right)$.

2. If $\varphi \in \mathcal{G}_{\Phi}$, then the condition (4.5) is necessary for the boundedness of $I_{\alpha}$ from $\mathcal{M}^{\Phi, \varphi}\left(\mathbb{R}^{n}\right)$ to $W \mathcal{M}^{\Psi, \eta}\left(\mathbb{R}^{n}\right)$.

3. Let $\varphi(t)$ satisfy (2.3). If $\varphi \in \mathcal{G}_{\Phi}$ satisfies the regularity condition (4.6), then the condition (4.5) is necessary and sufficient for the boundedness of $I_{\alpha}$ from $\mathcal{M}^{\Phi, \varphi}\left(\mathbb{R}^{n}\right)$ to $W \mathcal{M}^{\Psi, \eta}\left(\mathbb{R}^{n}\right)$. 
Proof The first part of the theorem is a corollary of Theorem 7.12.

We shall now prove the second part. Let $B_{0}=B\left(x_{0}, t_{0}\right)$ and $x \in B_{0}$. By Lemma 3.3 we have $t_{0}^{\alpha} \leq C I_{\alpha} \chi_{B_{0}}(x)$. Therefore, by Lemmas 7.3 and 2.7

$$
\begin{aligned}
t_{0}^{\alpha} & \leq C \Psi^{-1}\left(\left|B_{0}\right|^{-1}\right)\left\|I_{\alpha} \chi_{B_{0}}\right\|_{W L^{\Psi}\left(B_{0}\right)} \leq C \eta\left(t_{0}\right)\left\|I_{\alpha} \chi_{B_{0}}\right\|_{W \mathcal{M}^{\Psi, \eta}} \\
& \leq C \eta\left(t_{0}\right)\left\|\chi_{B_{0}}\right\|_{\mathcal{M}^{\Phi, \varphi}} \leq C \frac{\eta\left(t_{0}\right)}{\varphi\left(t_{0}\right)}=C \varphi\left(t_{0}\right)^{\beta-1} .
\end{aligned}
$$

Since this is true for every $t_{0}>0$, we are done.

The third statement of the theorem follows from the first and second parts of the theorem.

If we take $\Phi(t)=t^{p}, p \in[1, \infty)$ and $\beta=\frac{p}{q}$ with $p<q<\infty$ at Theorem 7.13 we get the following new result for generalized Morrey spaces.

Corollary 7.14 Let $1 \leq p<q<\infty$.

1. If $\varphi(t)$ satisfies (1.1), then the condition (4.7) is sufficient for the boundedness of $I_{\alpha}$ from $\mathcal{M}^{p, \varphi}\left(\mathbb{R}^{n}\right)$ to $W \mathcal{M}^{q, \varphi^{\frac{p}{q}}}\left(\mathbb{R}^{n}\right)$.

2. If $\varphi \in \mathcal{G}_{p}$, then the condition (4.8) is necessary for the boundedness of $I_{\alpha}$ from $\mathcal{M}^{p, \varphi}\left(\mathbb{R}^{n}\right)$ to $W \mathcal{M}^{q, \varphi^{\frac{p}{q}}}\left(\mathbb{R}^{n}\right)$.

3. If $\varphi \in \mathcal{G}_{p}$ satisfies the regularity condition (4.6), then the condition (4.8) is necessary and sufficient for the boundedness of $I_{\alpha}$ from $\mathcal{M}^{p, \varphi}\left(\mathbb{R}^{n}\right)$ to $W \mathcal{M}^{q, \varphi^{\frac{p}{q}}}\left(\mathbb{R}^{n}\right)$.

Remark 7.15 If we take into account Remark 4.5 we get the following weak version of Adams result for Morrey spaces.

Corollary 7.16 Let $0<\alpha<n, 1 \leq p<q<\infty$ and $0<\lambda<n-\alpha p$. Then $I_{\alpha}$ is bounded from $\mathcal{M}^{p, \lambda}\left(\mathbb{R}^{n}\right)$ to $W \mathcal{M}^{q, \lambda}\left(\mathbb{R}^{n}\right)$ if and only if $\frac{1}{p}-\frac{1}{q}=\frac{\alpha}{n-\lambda}$.

\section{Competing interests}

The authors declare that they have no competing interests.

\section{Authors' contributions}

This work was carried out in collaboration between all authors. VSG raised these interesting problems in the research. VSG, FD, and SGH proved the theorems, interpreted the results, and wrote the article. All authors defined the research theme, read, and approved the manuscript.

\section{Author details}

'Department of Mathematics, Ahi Evran University, Kirsehir, Turkey. ${ }^{2}$ Institute of Mathematics and Mechanics of NAS of Azerbaijan, Baku, Azerbaijan. ${ }^{3}$ Ganja State University, Ganja, Azerbaijan.

\section{Acknowledgements}

We thank the referee(s) for careful reading the paper and useful comments. The research of VS Guliyev and F Deringoz was partially supported by the grant of Ahi Evran University Scientific Research Project (FEF.A3.16.024).

Received: 7 September 2016 Accepted: 30 September 2016 Published online: 11 October 2016

\section{References}

1. Guliyev, VS: Boundedness of the maximal, potential and singular operators in the generalized Morrey spaces. J. Inequal. Appl. 2009, Article ID 503948 (2009)

2. Nakai, E: A characterization of pointwise multipliers on the Morrey spaces. Sci. Math. 3, $445-454$ (2000)

3. Persson, L-E, Ragusa, MA, Samko, N, Wall, P: Commutators of Hardy operators in vanishing Morrey spaces. In: 9th Int. Conf. on Math. Problems in Engineering, Aerospace and Sciences (ICNPAA 2012). AIP Conf. Proc., vol. 1493, pp. 859-866 (2012) 
4. Orlicz, W: Über eine gewisse Klasse von Räumen vom Typus B. Bull. Acad. Pol. A, $207-220$ (1932); reprinted in: Collected Papers, PWN, Warszawa, 217-230 (1988)

5. Orlicz, W: Über Räume (LM $)$. Bull. Acad. Pol. A, 93-107 (1936); reprinted in: Collected Papers, PWN, Warszawa, 345-359 (1988)

6. Kita, H: On maximal functions in Orlicz spaces. Proc. Am. Math. Soc. 124, 3019-3025 (1996)

7. Kita, H: On Hardy-Littlewood maximal functions in Orlicz spaces. Math. Nachr. 183, 135-155 (1997)

8. Cianchi, A: Strong and weak type inequalities for some classical operators in Orlicz spaces. J. Lond. Math. Soc. 60(2), 187-202 (1999)

9. Nakai, E: Generalized fractional integrals on Orlicz-Morrey spaces. In: Banach and Function Spaces (Kitakyushu, 2003), pp. 323-333. Yokohama Publishers, Yokohama (2004)

10. Sawano, Y, Sugano, S, Tanaka, H: Orlicz-Morrey spaces and fractional operators. Potential Anal. 36(4), 517-556 (2012)

11. Deringoz, F, Guliyev, VS, Samko, S: Boundedness of maximal and singular operators on generalized Orlicz-Morrey spaces. In: Operator Theory, Operator Algebras and Applications. Operator Theory: Advances and Applications, vol. 242, pp. 139-158 (2014)

12. Peetre, J: On the theory of $M_{p \lambda}$. J. Funct. Anal. 4, 71-87 (1969)

13. Adams, DR: A note on Riesz potentials. Duke Math. J. 42, 765-778 (1975)

14. Chiarenza, F, Frasca, M: Morrey spaces and Hardy-Littlewood maximal function. Rend. Mat. Appl. (7) 7, 273-279 (1987)

15. Guliyev, VS: Integral operators on function spaces on the homogeneous groups and on domains in $\mathbb{R}^{n}$. Doctor's degree dissertation, Mat. Inst. Steklov, Moscow (1994) (in Russian)

16. Guliyev, VS: Function Spaces, Integral Operators and Two Weighted Inequalities on Homogeneous Groups: Some Applications. Casioglu, Baku (1999) (in Russian)

17. Guliyev, VS, Aliyev, SS, Karaman, T, Shukurov, PS: Boundedness of sublinear operators and commutators on generalized Morrey space. Integral Equ. Oper. Theory 71(3), 327-355 (2011)

18. Guliyev, VS, Shukurov, PS: On the boundedness of the fractional maximal operator, Riesz potential and their commutators in generalized Morrey spaces. In: Advances in Harmonic Analysis and Operator Theory. Operator Theory: Advances and Applications, vol. 229, pp. 175-194 (2013)

19. Guliyev, VS, Deringoz, F: On the Riesz potential and its commutators on generalized Orlicz-Morrey spaces. J. Funct. Spaces 2014, Article ID 617414 (2014)

20. Ragusa, MA: Necessary and sufficient condition for a VMO function. Appl. Math. Comput. 218, 11952-11958 (2012)

21. Hedberg, LI: On certain convolution inequalities. Proc. Am. Math. Soc. 36, 505-510 (1972)

22. Janson, S: Mean oscillation and commutators of singular integral operators. Ark. Mat. 16, 263-270 (1978)

23. Ho, K-P: Characterization of BMO in terms of rearrangement-invariant Banach function spaces. Expo. Math. 27, 363-372 (2009)

24. Deringoz, F, Guliyev, VS, Samko, S: Boundedness of the maximal operator and its commutators on vanishing generalized Orlicz-Morrey spaces. Ann. Acad. Sci. Fenn., Math. 40, 535-549 (2015)

\section{Submit your manuscript to a SpringerOpen ${ }^{\circledR}$ journal and benefit from:}

- Convenient online submission

- Rigorous peer review

- Immediate publication on acceptance

Open access: articles freely available online

- High visibility within the field

- Retaining the copyright to your article 